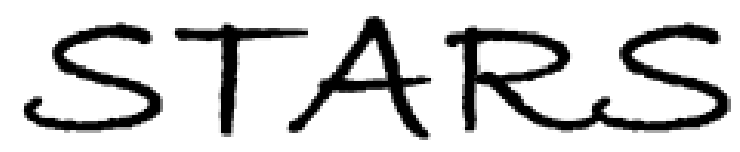

University of Central Florida

STARS

$1-1-2012$

\title{
Surface depletion mediated control of inter-sub-band absorption in GaAs/AlAs semiconductor quantum well systems
}

Walter R. Buchwald

University of Central Florida

Justin W. Cleary

Joshua Hendrickson

Find similar works at: https://stars.library.ucf.edu/facultybib2010

University of Central Florida Libraries http://library.ucf.edu

This Article is brought to you for free and open access by the Faculty Bibliography at STARS. It has been accepted for inclusion in Faculty Bibliography 2010 s by an authorized administrator of STARS. For more information, please contactSTARS@ucf.edu.

\section{Recommended Citation}

Buchwald, Walter R.; Cleary, Justin W.; and Hendrickson, Joshua, "Surface depletion mediated control of inter-sub-band absorption in GaAs/AIAs semiconductor quantum well systems" (2012). Faculty Bibliography 2010s. 2338.

https://stars.library.ucf.edu/facultybib2010/2338

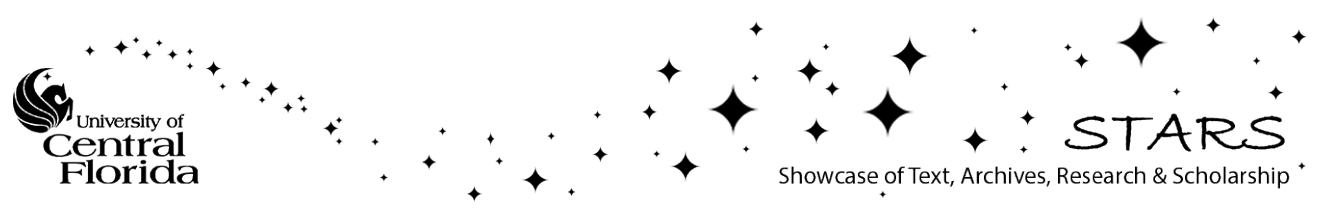




\section{Surface depletion mediated control of inter-sub-band absorption in GaAs/AIAs semiconductor quantum well systems}

Cite as: Appl. Phys. Lett. 100, 051110 (2012); https://doi.org/10.1063/1.3680232

Submitted: 21 November 2011 . Accepted: 04 January 2012. Published Online: 02 February 2012

Walter R. Buchwald, Justin W. Cleary, and Joshua Hendrickson

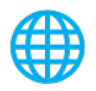

\section{Applied Physics Reviews} Now accepting original research 


\title{
Surface depletion mediated control of inter-sub-band absorption in GaAs/AIAs semiconductor quantum well systems
}

\author{
Walter R. Buchwald, ${ }^{1, a)}$ Justin W. Cleary, ${ }^{2}$ and Joshua Hendrickson ${ }^{2}$ \\ ${ }^{1}$ Solid State Scientific Corporation, Nashua, New Hampshire 03060, USA \\ and Physics Department, University of Central Florida, Orlando, Florida 32816, USA \\ ${ }^{2}$ Air Force Research Laboratory, Sensors Directorate, Wright Paterson AFB, Ohio 45433, USA
}

(Received 21 November 2011; accepted 4 January 2012; published online 2 February 2012)

The modification of quantum well inter-sub-band absorption properties due to surface depletion induced band bending is reported. Fourier transform infrared spectroscopy measurements of a GaAs/AlAs multiple quantum well system reveal a reduction in the characteristic absorption resonance in correlation with wet chemical etching. High resolution transmission electron microscopy confirms the presence of the quantum wells after etching, suggesting the quantum wells are positioned within the surface depletion region of the structure. This method of inter-sub-band absorption modification could be used for the formation of quantum dots from a quantum well system with the precise, deterministic control of their location. (c) 2012 American Institute of Physics. [doi:10.1063/1.3680232]

Although the traditional Stranski-Krastanow (S-K) method of quantum dot growth is known to produce high quality three dimensional electron confinement, dot to dot variations in bound state transition energies and the difficulties with the deterministic placement of these dots have historically motivated research into other methods of quantum dot formation. For example, the etch induced modification of a quantum well (QW) system to form a mesa structure has been shown to produce the required three dimensional confinement, however, this method typically requires the addition of surface overgrowth techniques to passivate any etch induced surface damage. ${ }^{1}$ Another method to obtain lateral confinement in a quantum well system is through the use of an externally applied bias voltage. ${ }^{2}$ This method gives external voltage control of the charging of the quantum dot but at the expense of a complicated fabrication procedure. In both cases, the quality of the dot when compared to the traditional $\mathrm{S}-\mathrm{K}$ growth method is somewhat compromised in favor of the precise, deterministic placement of the quantum dot on the semiconductor surface.

This work reports a method that will provide lateral confinement in a QW system, effectively making the system three dimensionally confined, without the detrimental effects associated with etching completely through the twodimensional electron gas, or, the need for a complex pattern of bias electrodes. This method, which relies on the inherent surface depletion associated with III-V semiconductors, is illustrated in Figure 1(a). ${ }^{3,4}$ This figure presents the conduction band profile of a GaAs cap layer, $0.2 \mu \mathrm{m}$ thick and doped n-type to $3.0 \times 10^{17} \mathrm{~cm}^{-3}$, followed by a GaAs spacer region, in this case $60 \mathrm{~nm}$ thick and doped n-type to $3.0 \times 10^{16} \mathrm{~cm}^{-3}$. The GaAs/AlAs QW containing layers follow and are located $0.26 \mu \mathrm{m}$ below the surface. The center of the QW is GaAs and doped n-type to $6 \times 10^{17} \mathrm{~cm}^{-3}$. In the figure, the dotted line represents the conduction band profile with the doped cap layer in place, and the solid line represents the conduction band profile with the cap layer

${ }^{a}$ Electronic mail: walter.buchwald@solidstatescientific.com. removed. In general, surface related defects cause the Fermi level to be pinned at roughly mid-gap inducing a built in potential of approximately $0.71 \mathrm{eV}$, which, in order for charge neutrality to be maintained, will cause the surface to be depleted of free carriers. ${ }^{5}$ The extent of this surface depletion layer will be determined by the doping concentration associated with the underlying material. Referring to Figure 1(a), when the higher doped cap layer is in place, the depletion width extends roughly $0.1 \mu \mathrm{m}$ from the surface. This positions the QW beyond the surface depletion width, and the ground state energy level associated with the QW will be filled with electrons. When the cap layer is removed, the surface depletion region will be defined by the lighter doping of the spacer and QW regions and will extend further from surface. This extended depletion width now places the QW in the surface depleted region and causes the ground state energy to have a greater energy separation with respect to the Fermi level which empty's the ground state of electrons and suppresses any inter-sub-band absorption. Further, relative to those locations where the cap layer is not removed, there exists a potential barrier, in Figure 1(a) seen to be roughly $0.20 \mathrm{eV}$, which is available to provide the needed lateral confinement to convert the $\mathrm{QW}$ system into a quantum dot (QDot) system. The lateral confinement provided using this method is a function of the spacer thickness as shown in Figure 1(b), which plots the difference between the barrier height of Figure 1(a) and the ground state energy level associated with the QW. As the spacer thickness is reduced, the lateral barrier height increases as expected. If the spacer thickness is too great, the barrier height becomes less than the ground state energy of the QW and no lateral confinement is obtained as seen in the figure for a spacer thickness greater than roughly $65 \mathrm{~nm}$.

The focus of this work is based on the notion that the suppression of an inter-sub-band absorption feature in a quantum well system can serve as a signature for the surface depletion control of the QW charge state provided an alternate means can be used to confirm the existence of the QW's after etching is complete. Here we utilize high resolution 

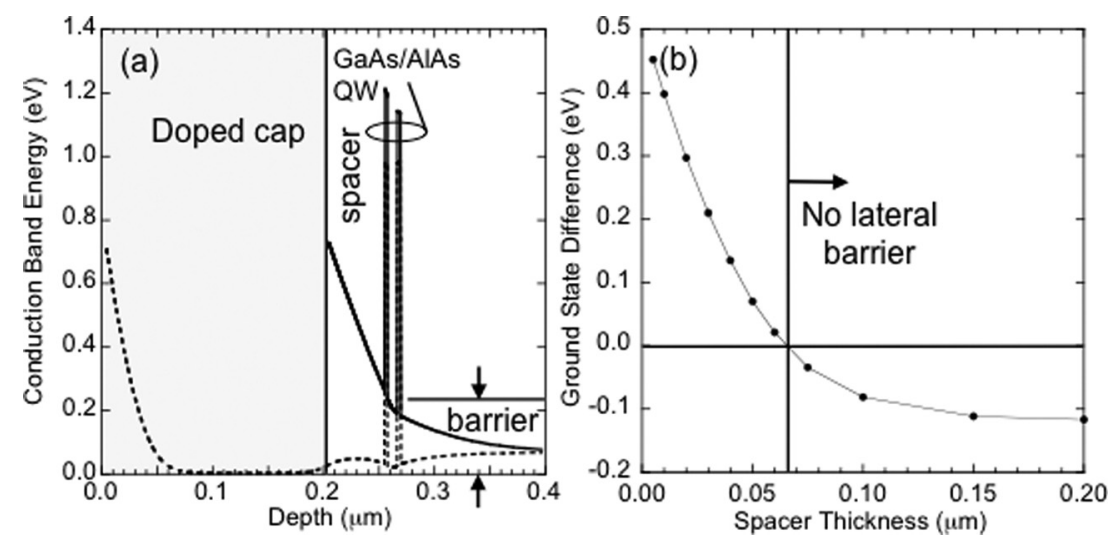

FIG. 1. (a) Conduction band energy diagram illustrating the use of surface depletion for control of QW charge state. Dotted and solid curves represent conduction band profiles with and without the doped cap in place. (b) Energy difference between the barrier height obtained using this method and the ground state energy of the QW as a function of spacer thickness.

transmission electron microscopy (TEM) cross sectional analysis of the etched sample to confirm the existence of the QW's while using fourier transform infrared spectroscopy (FTIR) measurements to monitor the inter-sub-band absorption feature. A clear reduction in the FTIR signal is observed which correlates well with the etch induced introduction of the QW's to the depletion region suggesting the usefulness of this method for inter-sub-band charge state control.

The samples used in this work were obtained commercially and consisted of a semi-insulating GaAs substrate on which was grown $2000 \AA$ of un-doped AlAs, $10000 \AA$ of GaAs $\left(3 \times 10^{18} \mathrm{~cm}^{-3} \mathrm{Si}\right), 30 \AA$ of un-doped AlAs followed by a 50 period QW stack with each period consisting of $10 \AA$ of un-doped GaAs, $63 \AA$ of GaAs $\left(6.0 \times 10^{17} \mathrm{~cm}^{-3}, \mathrm{Si}\right), 10 \AA$ of un-doped GaAs, and $30 \AA$ of un-doped AlAs. This structure was capped with a single $5000 \AA$ layer of $\mathrm{GaAs}$ $\left(3.0 \times 10^{18} \mathrm{~cm}^{-3}, \mathrm{Si}\right)$. To determine the bound state intersub-band absorption energy, a Bruker V80V FTIR spectrometer coupled to a Hyperion FTIR microscope with a $\mathrm{LN}_{2}$ cooled MCT detector was used. To maximize the FTIR signal a roughly $1 \mathrm{~cm} \times 1 \mathrm{~cm}$ sample was angle polished to $45^{\circ}$, held vertically between the condenser lenses of the FTIR microscope, and illuminated through the angle polished end facet. Incident radiation polarized normal to the material

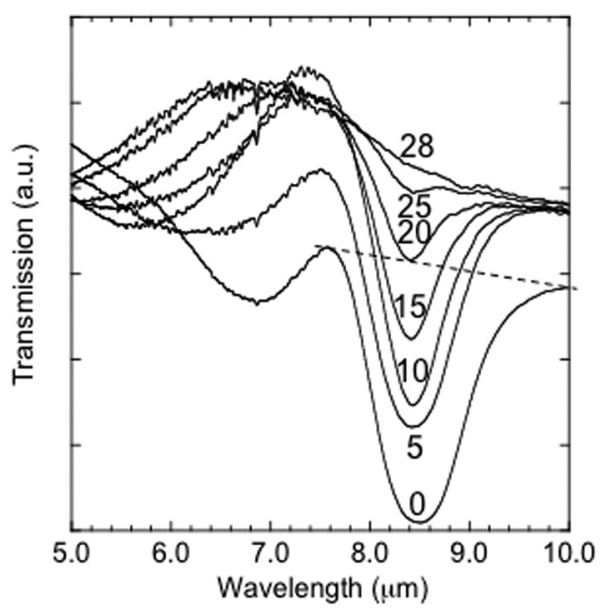

FIG. 2. FTIR transmission data showing $8.4 \mu \mathrm{m}$ ground to first excited state quantum well absorption feature. Numbers on each curve represent total $\mathrm{NH}_{4} \mathrm{OH}: \mathrm{H}_{2} \mathrm{O}_{2}: \mathrm{H}_{2} \mathrm{O}$ etch time. Raw data was shifted vertically to produce equal average transmission relative to large Fabry-Perot oscillations in the $2 \mu \mathrm{m}$ to $4 \mu \mathrm{m}$ wavelength range. Dotted line represents baseline subtraction method used to determine peak height. surface was background corrected with radiation polarized in the plane of the material surface to obtain the desired intersub-band spectral response. ${ }^{6}$

Etching of the sample was undertaken using a mixture of $\mathrm{NH}_{4} \mathrm{OH}: \mathrm{H}_{2} \mathrm{O}_{2}: \mathrm{H}_{2} \mathrm{O}(2: 1: 10)$, reported to selectively etch GaAs over AlAs, and a $10 \%$ mixture of $\mathrm{HF}$, reported to selectively etch AlAs over GaAs. ${ }^{7}$ The procedure followed was to place the sample in the $\mathrm{NH}_{4} \mathrm{OH}: \mathrm{H}_{2} \mathrm{O}_{2}: \mathrm{H}_{2} \mathrm{O}$ solution for $5 \mathrm{~s}$ followed by $10 \mathrm{~s}$ in the $10 \% \mathrm{HF}$ solution. After rinsing and drying the sample was immediately measured in the FTIR. This sequence of etch and measure was completed six times and, as will be shown, corresponded to a $90 \%$ reduction in the inter-sub-band absorption feature. A final etch of $3 \mathrm{~s}$ in both solutions reduced the FTIR signature even further to $98 \%$ of its original value. After the final etch the sample was cross sectioned with a focused ion beam and high resolution TEM was used to confirm the presence of any remaining quantum wells.

Figure 2 presents FTIR transmission spectra of this material obtained after each subsequent etch step, as described earlier. A clear, polarization dependent absorption is seen at a wavelength of $8.4 \mu \mathrm{m}$, which represents the ground to first excited state inter-sub-band transition. This absorption feature is observed to decrease in intensity as the etch cycles commence. In order to take into account slight variations in the overall optical transmission through the sample, all curves of Figure 2 were shifted vertically in order to align the average transmission in the $2 \mu \mathrm{m}$ to $4 \mu \mathrm{m}$ region of the spectrum with each other. The dotted line, drawn between

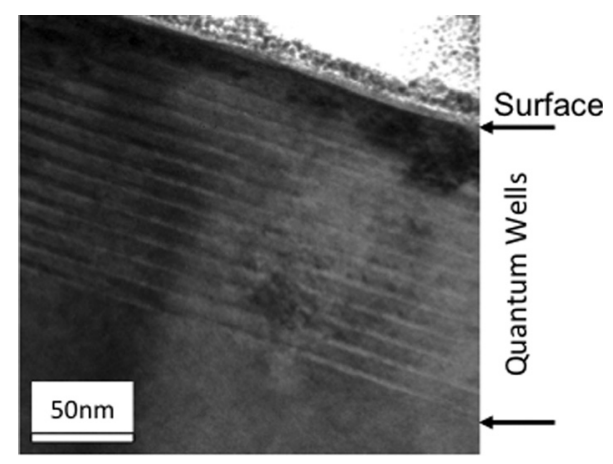

FIG. 3. TEM image of cross-sectioned epi-layer. 12 quantum wells are observed indicating surface depletion control of the QW ground state electron occupancy causing a reduction in the characteristic QW FTIR absorption feature. 


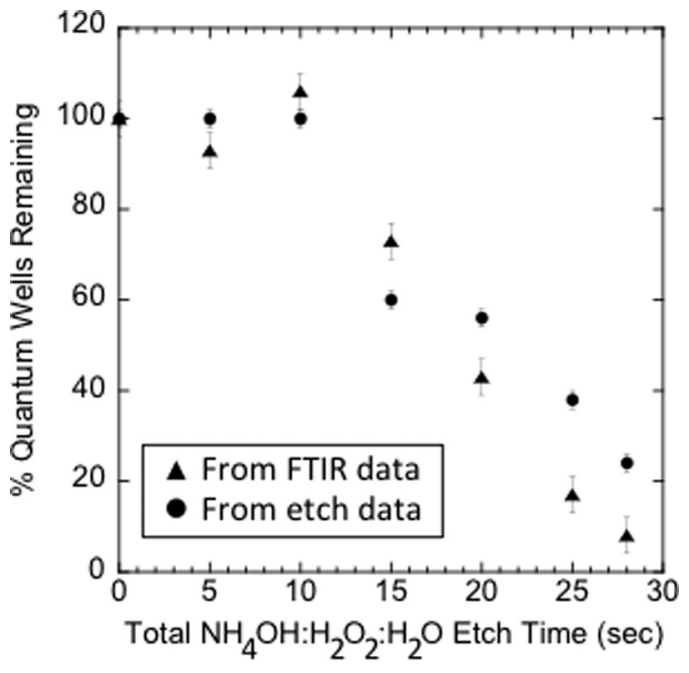

FIG. 4. A comparison between the QW removal rate as determined by step profilometer measurments of the etched sample and the reduction in FTIR signal caused by the etching.

the shoulders of the characteristic absorption feature in this figure, represents the typical baseline subtraction method used to determine the actual peak heights in this work.

Figure 3 shows the cross sectional TEM of the sample obtained after the last etching sequence. The clear presence of quantum wells indicates the reduction in the characteristic absorption feature was not caused simply by the complete removal of the QW stack and suggests its cause is a depopulation of the ground state occupancy due to the QWs being located within the inherent surface depletion region associated with this material.

The final method used to validate the use of surface depletion for the charge state control of inter-sub-band quantum well absorption was to correlate the etch rate of the material with the rate of reduction of the characteristic FTIR absorption feature observed during the etch process. Material etch rate was determined using an identical, photolithographically defined sample and a step profilometer. This second sample was etched using the same etch sequence as described above. Using the TEM data to calibrate the etch depth, it was determined that the first two etch sequences were only sufficient to remove $0.45 \mu \mathrm{m}$ of the cap layer. Therefore, taking into account the potential for surface depletion within the remaining $0.05 \mu \mathrm{m}$ of the cap layer, the curve labeled "10" in Figure 2 represents all 50 QW's still in place with the cap layer removed. Figure 4 plots the percentage of quantum wells remaining obtained independently using both the etch data and the FTIR data. Good agreement is seen between the two rates, again suggesting surface depletion as a viable means to control inter-sub-band QW absorption. The slight wavelength shift associated with the curves labeled " 0 " and " 5 " in Figure 2 are most likely caused by interactions between the QW's and the waveguide-like structure used and are beyond the scope of this work. ${ }^{8}$

A method for the control of inter-sub-band absorption in QW's through the use of the surface depletion region inherent to compound semiconductors has been presented. It is suggested that this method warrants investigation as a means to provide improved three-dimensional confinement in inherently two-dimensional, or quantum well, confined systems. Although the method presented here suggests the etching of doped layers to modify the extent of the surface depletion layer, an alternate approach would be the selective ionimplantation of charge into un-doped layers to provide a similar result but with improved lateral dimensions. It is suggested that this approach could find usefulness in integrated quantum optic applications where the deterministic placement of three-dimensional quantum confined, atom-like states into small mode volume photonic crystal cavities is required. This, along with the potential for waveguide coupling, the monolithic integration of long wavelength quantum cascade laser sources, and integrated single photon detectors could lead to a class of integrated circuits exhibiting a quantum optics based functionality.

Support for this work is provided by AFOSR LRIR award number 09RY08COR (Gernot Pomrenke, PM).

${ }^{1}$ V. B. Verma, Martin J. Stevens, K. L. Silverman, N. L. Dias, A. Garg, J. J. Coleman, and R. P. Mirin, Opt. Express 19, 4182 (2011).

${ }^{2}$ C. Rossler, M. Bichler, D. Schuh, W. Wegscheider, and S. Ludwig, Nanotechnology 19, 165201 (2008).

${ }^{3}$ J. Bardeen, Phys. Rev. 71, 717 (1947).

${ }^{4}$ J. Tarsoff, Phys. Rev. B 32, 6968 (1985).

${ }^{5}$ M. Murakami, Sci. Technol. Adv. Mater. 3, 1 (2002).

${ }^{6}$ B. F. Levine, R. J. Malik, J. Walker, K. K. Choi, C. G. Bethea, D. A. Kleinman, and J. M. Vandenberg, Appl. Phys. Lett, 50, 273 (1987).

${ }^{7}$ J. Voncken, J. J. Schermer, G. J. Bauhuis, T. J. van Niftrik, and P. K. Larsen, J. Phys.: Condens. Matter 16, 3585 (2004).

${ }^{8}$ D. Dini, R. Kohler, A. Tredicucci, G. Biasiol, and L. Sorba, Phys. Rev. Lett. 90, 116401 (2003). 